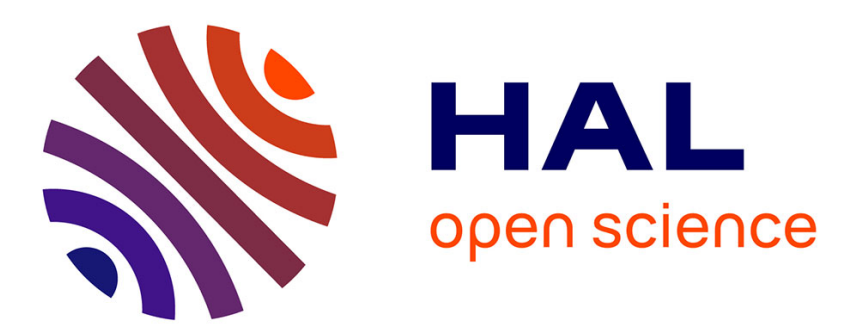

\title{
Robust Adaptive Extended Kalman Filtering for Real Time MR-Thermometry Guided HIFU Interventions
}

Sébastien Roujol, Baudouin Denis de Senneville, Silke Hey, Chrit Moonen, Mario Ries

\section{- To cite this version:}

Sébastien Roujol, Baudouin Denis de Senneville, Silke Hey, Chrit Moonen, Mario Ries. Robust Adaptive Extended Kalman Filtering for Real Time MR-Thermometry Guided HIFU Interventions. IEEE Transactions on Medical Imaging, 2012, 31 (3), pp.533-542. 10.1109/TMI.2011.2171772 . hal01578186

\author{
HAL Id: hal-01578186 \\ https://hal.science/hal-01578186
}

Submitted on 28 Aug 2017

HAL is a multi-disciplinary open access archive for the deposit and dissemination of scientific research documents, whether they are published or not. The documents may come from teaching and research institutions in France or abroad, or from public or private research centers.
L'archive ouverte pluridisciplinaire HAL, est destinée au dépôt et à la diffusion de documents scientifiques de niveau recherche, publiés ou non, émanant des établissements d'enseignement et de recherche français ou étrangers, des laboratoires publics ou privés. 


\title{
Robust adaptive extended Kalman filtering for real time MR-thermometry guided HIFU interventions
}

\author{
Sébastien Roujol, Baudouin Denis de Senneville, Silke Hey, Chrit Moonen, Mario Ries
}

\begin{abstract}
Real time magnetic resonance (MR) thermometry is gaining clinical importance for monitoring and guiding high intensity focused ultrasound (HIFU) ablations of tumorous tissue. The temperature information can be employed to adjust the position and the power of the HIFU system in real time and to determine the therapy endpoint.

The requirement to resove both physiological motion on mobile organs and the rapid temperature variations induced by state-ofthe art high-power HIFU systems requires fast MRI-acquisition schemes, which are generally hampered by low signal to noise ratios (SNR). This directly limits the precision of real time MR-thermometry and thus in many cases the feasability of sophisticated control algorithms. To overcome these limitations, temporal filtering of the temperature has been suggested in the past, which has generally an adverse impact on the accuracy and latency of the filtered data.

Here, we propose a novel model based digital filter combining an extended Kalman filter (EKF) with a predictive model of the temperature based on the bio heat transfer equation. This filter aims to improve the precision of MR-thermometry while monitoring and adapting its impact on the accuracy using the formalism of adaptive extended Kalman filtering. An additional outlier rejection addresses the problem of sparse artifacted temperature points. The filter was evaluated and compared to a matched FIR filter using simulated data, HIFU experiments on phantoms and in vivo data obtained during HIFU ablations on porcine kidneys. The filter provides improved artefact and noise reduction, while having a minimal impact on accuracy and latency.
\end{abstract}

Index Terms-Biomedical signal processing, Kalman filters, Real time systems, Magnetic resonance imaging.

\section{INTRODUCTION}

Recently, MR-thermometry has evolved to the method of choice for monitoring and guiding thermal therapies such as radio-frequency [1], laser [2] or HIFU thermal ablation [3]. The availability in real time of temperature information is required for several aspects of the therapeutic intervention. Temperature maps can be used for retro active feedback control of the ablation device. For the particular case of non-invasive HIFU ablations, volumetric heating strategies [4]

Manuscript received XX XX, 2010; This work was supported in part by Ligue Nationale Contre le Cancer, Conseil Régional d'Aquitaine, Diagnostic Molecular Imaging, Agence National de Recherche, Fondation InNaBioSanté and Philips Medical System. Asterisk indicates the corresponding author.

M. Roujol, Denis de Senneville, Moonen and Ries are with the Laboratory for Molecular and Functional Imaging: from Physiology to Therapy FRE 3313 CNRS/ University Victor Segalen Bordeaux 146 rue Leo Saignat, Case 11733076 Bordeaux, France. e-mail: sebastien.roujol@imf.ubordeaux2.fr; baudouin@imf.u-bordeaux2.fr;silke.hey@imf.u-bordeaux2.fr chrit.moonen@imf.u-bordeaux2.fr;mario.ries@imf.u-bordeaux2.fr

M. Roujol is also with Laboratoire Bordelais de Recherche en Informatique, UMR 5800 CNRS/University of Bordeaux 1 - 351, cours de la Liberation, 33405 Talence, France adapt the position of the focal point and the delivered power based on past measured temperature evolution. In addition, the evolution of the temperature $(T(t)$, with $t$ the time) can be used to obtain the thermal dose $(T D)$ as follows:

$$
T D=\left\{\begin{array}{l}
\int_{0}^{t} 2^{T(t)-43}, \text { if } T<43^{\circ} C \\
\int_{0}^{t} 4^{T(t)-43}, \text { if } T>43^{\circ} C
\end{array}\right.
$$

which provides an accurate and immediate prediction of tissue necrosis [5], which in turn allows to determine the therapy endpoint.

However, the precision of real time MR-thermometry is generally limited by both the available SNR [6] and the influence of physiological motion. Consequently, algorithms used for retroactive control are liable to be biased or rendered unstable by the presence of noise on the temperature maps. Furthermore, the accuracy of the calculation of the thermal dose used for necrosis estimation deteriorates rapidly for low SNRs due to its exponential dependence on the temperature. To overcome these problems, temporal filtering has been proposed as a solution to improve the precision of the temperature maps using an infinite impulse response (IIR) filter [7]. However, the use of this type of low-pass filters, or alternative designs such as finite impulse response (FIR) filters, introduces in general additional latency, leading to a reduced accuracy and limits the achievable temporal resolution of the observation process.

This can be alleviated by using more complex filter designs, which include physical knowledge of the observed system, such as Kalman filters [8]. Kalman filtering is based on the combination of both measured data and data derived from a forecast based on a physical model. Potocki \& Tharp in [9] proposed the bio heat transfer equation (BHTE) model [10] for this purpose. In their experiment, temperature information was only available at four different locations (obtained from optical fibers). The filter was essentially not designed for noise removal (since only four measurement points were available) but to estimate both blood perfusion and temperature information at unmeasured locations (spatial extrapolation). More recently, Ye et al [11] employed Kalman filtering for improved model-based ultrasound temperature visualization. The employed predictor model was based on the construction of isothermal ellipsoids around the heated area. Although the precision of the resulting temperature maps was clearly improved (due to a high confidence placed in the model), the accuracy of the method was not evaluated.

Here, we propose a novel spatio-temporal filter based on the Kalman filtering theory that aims to improve MR-thermometry 
precision while controlling its accuracy. For this, an extended Kalman filter is employed with the BHTE as a predictive model. However, the combination of the predicted data and measured data is not a simple problem. A too large influence of the model would lead to a high noise reduction, but may introduce severe bias on the output accuracy if the model is not properly configured. With increasing emphasis on measured data, the noise removal will not be efficient, however the filter will tolerate imprecise calibration without giving rise to systematic error. Therefore, the filter has to be tuned in function of the model accuracy which is a priori not known and may vary in time. Therefore a dynamic evaluation of the model accuracy was added to the filtering process in order to adjust in real time the confidence in the model and thus the balance between measured and predicted data. The proposed filter was evaluated on simulated datasets and its feasibility is demonstrated on MR-guided HIFU experiments on a agarose gel phantom and in-vivo on a porcine kidney. It showed significant improvement compared to non-adaptive temporal filter designs without giving rise to additional filtering latency.

\section{MATERIAL AND METHODS}

\section{A. Temperature modeling using the Bio Heat Transfer Equa- tion (BHTE) model}

The BHTE model can be used to predict the temperature $T$ from time $t-1$ to time $t$, based on the applied acoustic power $P$ and a priori knowledge of the absorption rate $(\alpha)$, the heat diffusion coefficient $(D)$ and the perfusion value $(w)$ [10]. Note that this simplified model assumes these coefficients as spatialy and temporally invariant. The BHTE in the voxel of coordinates $\vec{r}=(x, y, z)$ is defined as follows:

$$
\frac{\partial}{\partial t} T_{(\vec{r}, t)}=\alpha \cdot P_{(\vec{r}, t)}+D \cdot \nabla T_{(\vec{r}, t)}-w \cdot T_{(\vec{r}, t)}
$$

where $\nabla$ denotes the lorentzian operator. In the absence of large vessels, perfusion effects are often neglected and thus $w$ is set to 0. The BHTE is generally solved in the Fourier domain since the problem can be turned into a linear differential equation as follows:

$$
\frac{\partial}{\partial t} \widetilde{T}_{(\vec{k}, t)}+\left(D \cdot k^{2}+w\right) \cdot \widetilde{T}_{(\vec{k}, t)}=\alpha \cdot \widetilde{P}_{(\vec{k}, t)}
$$

where $\widetilde{T}, \widetilde{P}$ denotes the Fourier transform of $T$ and $P$ respectively and $k$ denotes the frequency coordinates in the Fourier domain. The solution is computed based on the variation of constant as follows:

$$
\widetilde{T}_{(\vec{k}, t)}=\widetilde{T}_{(\vec{k}, t-1)} \cdot e^{-\left(D \cdot k^{2}+w\right) t}+\alpha \cdot \widetilde{P}_{(\vec{k}, t)} \frac{1-e^{-\left(D \cdot k^{2}+w\right) t}}{D \cdot k^{2}+w}
$$

Since the BHTE models the physical processes of heat diffusion and absorption, it requires to be applied in 3D space in order to obtain unbiased results. Unfortunately, the available acquisition time in is typically too limited in interventional imaging to obtain full 3D temperature imaging, especially if physiological motion has to be resolved. As a consequence, the BHTE has in practice often to be applied to $2 \mathrm{D}$ or severely undersampled 3D datasets. This leads to a systematic underestimation of the heat evacuation and neglects potential heat inflow from adjacent slices, in particular for large $\Delta t$.In order to evaluate the resulting bias on the filtered temperature data, the BHTE was implemented for both 2D and 3D temperature prediction and the results subsequently compared on simulated heating experiments.

Furthermore, although MR-thermometry provides coherent temperature information in areas of high signal (generally with a precision of few degrees), areas with very low signal level generally display randomized temperature values (in the range of several dozens of degrees of erroneous variations). Since the BHTE model prediction is based on preceding filtered temperature maps represented in Fourier space, such areas have to be excluded by manual ROI-based masking to prevent a bias due to undesired high frequency noise.

\section{B. BHTE based extended Kalman filtering}

Since the discrete solution of the BHTE represents a nonlinear model for data prediction, the original Kalman filtering theory, which requires a linear predictor, is not directly applicable. Non-linear predictors are addressed by the extendend Kalman filtering (EKF) formalism. An EKF can be seen as a two step process. In a first pass, the filter computes a data prediction (at time $t$ ) based on the last filtered data point (obtained at time $t-1$ ). In a second pass, the algorithm optimally combines both predicted data and measured data to obtain the final filtered data corresponding to time $t$.

1) First pass: time update equations: The first pass of the filter is often referred to as time update equations. The temperature prediction $T_{t}^{-}$at time $t$ and the a priori estimate error covariance $P_{t}^{-}$are computed as follows:

$$
\begin{aligned}
& T_{t}^{-}=f\left(T_{t-1}, u_{t-1}\right) \\
& P_{t}^{-}=A_{t} P_{t-1} A_{t}^{T}+Q
\end{aligned}
$$

where $T_{t}^{-}$denotes the model prediction at time $t, T_{t-1}$ the filtered temperature at time $t-1, f$ represents the BHTE model, $u_{t-1}$ is the control input parameter which in our case corresponds to the HIFU delivered power at time $t-1$. $A_{t}$ is the Jacobian matrix of partial derivatives of $f$ with respect to the temperature $T$ at $\left(T_{t-1}, u_{t-1}\right), A_{t}^{T}$ denotes the transpose of $A_{t}, P_{t-1}$ is the a posteriori estimate error covariance at time $t-1$ and $Q$ is the process noise covariance related to the model innacuracy.

2) Second pass: measurement update equations: In a second pass, the Kalman filter combines both predicted $T_{t}^{-}$and measured $T_{t}^{m}$ data to obtain the final filtered data $T_{t}$. The combination is weighted with a parameter $K_{t}$, often referred to as the Kalman gain, while the difference $T_{t}^{m}-H T_{t}^{-}$is called the innovation $S_{t}$. During the second pass, $K_{t}$ is first updated based on the new a priori estimate error covariance. Subsequently, the filtered temperature $T_{t}$ can be computed from the weighted innovation $S_{t}$. The final step updates the a posteriori estimate error covariance $P_{t}$. The measurement update equations are summarized as follows: 


$$
\begin{aligned}
& K_{t}=P_{t}^{-} H^{T}\left(H P_{t}^{-} H^{T}+R\right)^{-1} \\
& T_{t}=T_{t}^{-}+K_{t}\left(T_{t}^{m}-H T_{t}^{-}\right) \\
& P_{t}=\left(I-K_{t} H\right) P_{t}^{-}
\end{aligned}
$$

where $H$ relates the model state to the measurement (here $H$ is the identity since the measurement are directly obtained in state space) and $R$ is the measurement noise covariance that relates to the MR-thermometry precision of the measure.

Note that $R$ can be obtained prior to hyperthermia by evaluating the standard deviation of the measured temperature.

\section{Autocalibrated extended Kalman filtering (AEKF)}

The EKF combines measured and predicted data via the Kalman gain $K_{t}$, that allows to optimally adjust the confidence between the employed model and the measured data. The Kalman gain is updated dynamically and predominantly influenced by two input parameters of the filter: The measurement noise covariance $(R)$ and the process noise covariance $(Q)$. While the measurement noise covariance can be determined from baseline data in absence of heating, the determination of $Q$ is not straightforward. $Q$ corresponds to the model accuracy and thus depends on the accurate knowledge of the physical parameters of the tissue (absorption coefficient and heat diffusion), which are a-priory not exactly known and thus in general only available as rough estimates.

In addition, heat absorption only occurs during the heating period when the HIFU system delivers acoustic energy, while heat diffusion is present during both heating and cooling. Therefore, the BHTE model may have a varying performance over time if not properly configured (for example using an incorrect absorption coefficient with the true diffusion coefficient). In such a case, $Q$ would be better chosen as time variant.

This can be achieved using an adaptive EKF (AEKF), where $Q$ is automatically adjusted over time based on a dynamic evaluation of the model accuracy for each new measurement. In the proposed implementation this is based on the assumption that temperature noise is white noise around the real value. The model is considered accurate at time $t$ if $\varepsilon_{t}$, the spatio-temporal sum of the difference between predicted and measured data, is below a predefined error threshold $\varepsilon_{\text {threshold, }}$, with

$$
\varepsilon_{t}=\sum_{i=t-N}^{N} \sum_{(x, y) \in \varrho}\left(\hat{T}_{i}^{-}(x, y)-T_{i}^{m}(x, y)\right) .
$$

Here, the model is evaluated over the temporal window size $N$ and over a voxel perimeter $\varrho$ around the focal point, which is in the scope of this paper refered to as the spatial window size $M . \varepsilon_{\text {threshold }}$ is chosen as the maximum acceptable penality of the filtering process on the measurement accuracy.

\section{Robust approach of AEKF}

The computation of temperature maps requires an image processing pipeline, that can be rather complex especially in the case of MR-thermometry applied to mobile organs. Therefore, severe artifacts on temperature maps can be observed for a vaiety of reasons such as incorrect phase unwrapping or imperfect motion compensation. This type of error can have amplitudes much larger than the imprecision caused by low SNRs. Since the determination of the thermal dose corresponds to the integral over time of the temperature (see equation 1), temperature artifacts introduce a non reversible error bias on the thermal dose calculation. In addition, due to the exponential dependance on the temperature, even temporally sparse occurances of severe temperature artifacts lead frequently to an apparent thermal dose which is magnitudes off the true value. For HIFU interventions which use the thermal dose as a criteria for the therapy endpoint, this potentially directly affects the success of the intervention (overestimation) or patient safety (underestimation) and is thus highly undesireable. To detect such occurances, an outlier rejection based on the Chauvenet's criterion [12] was applied to the difference between measured and predicted temperature, i.e. the innovation $S_{t}$. The outlier rejection considers an innovation $S_{t}$ for rejection if the probability to obtain its deviation from the mean $\bar{S}_{t}$ is less than $1 /(2 \cdot N S), N S$ being the number of measurement samples. Substituting $\bar{S}_{t}$ by $\varepsilon_{t-1}$ as defined in equation 7 , leads to the following formulation of Chauvenet's criterion:

$$
T_{t}=\left\{\begin{array}{cl}
T_{t}^{-} & , \text {if }\left|S_{t}-\varepsilon_{t-1}\right|>\sigma\left(S_{t-1}\right) * e_{\max } \\
T_{t}^{-}+K_{t} S_{t} & \text {, if }\left|S_{t}-\varepsilon_{t-1}\right| \leq \sigma\left(S_{t-1}\right) * e_{\max }
\end{array}\right.
$$

where $e_{\max }$ is the ratio of maximum acceptable deviation to precision [12] and $\sigma\left(S_{t-1}\right)$ is the standard deviation of the innovation over the $N \times M$ sample points, excluding the last innovation $S_{t}$. Note that a rejection of a measured temperature value $T_{t}^{m}$ leads the filtered value $T_{t}$ to depend only on the prediction $T_{t}^{-}$, which is equivalent to temporarily setting $\mathrm{R}=\infty$ in equation 6 .

\section{E. Experimental set up}

1) Simulations: A $3 \mathrm{D}$ reference temperature dataset was simulated using the BHTE model (absorption $=0.02 \mathrm{KJ}^{-1}$, diffusion $=0.1 \mathrm{~mm}^{2} \mathrm{~s}^{-1}$, delivered power $=100 \mathrm{~W}$ (between $20^{t h}$ and $70^{\text {th }}$ dynamics), field of view $=32 \times 32 \times 16$, voxel size $=1 \times 1 \times 2 \mathrm{~mm}^{3}$, focal point size $=1.23 \times 1.23 \times 7.88$ $\mathrm{mm}^{3}$, dynamic scan time $\left.=1 \mathrm{~s}\right) . \mathrm{N}(=100)$ datasets were derived with added Gaussian noise $\left(\sigma=5^{\circ} \mathrm{C}\right)$. The proposed AEKF was evaluated using a 3D implementation of the filter. Since in practical cases, MR-temperature information is often available in $2 \mathrm{D}$, the impact of a $2 \mathrm{D}$ implementation of the filter was also analyzed.

A matched Kaiser Bessel FIR filter was used for comparison and configured to retain $90 \%$ of the integrated power spectrum of the original noise free temperature simulation (pass band cutoff frequency $=12 \mathrm{~Hz}$, stop-band attenuation $>21 \mathrm{db}$, window size=15).

To quantify the performances of each filter, the mean square error (MSE) between filter output and reference data was used as quality criterion of the filter output accuracy.

2) MR-guided HIFU experiments: MR-imaging was performed on a Philips Achieva 1.5 T MRI (Philips, Best, the Netherlands) and HIFU heating was performed using the integrated Philips' Sonalleve MR-HIFU system (Philips, Helsinki, Finland). 
a) Heating experiment on an agarose gel phantom: Dynamic MR-temperature imaging of an agarose gel phantom was obtained with a gradient recalled single-shot echo-planar sequence (300 images, single slice, $\mathrm{TR}=106 \mathrm{~ms}, \mathrm{TE}=36 \mathrm{~ms}$, flip angle $=40^{\circ}$, voxel size $=1.7 \times 1.7 \times 3 \mathrm{~mm}^{3}$, matrix $=64 \times 64$ ). HIFU heating protocol employed $50 \mathrm{~W}$ of electrical power to the HIFU transducer over a period of $40 \mathrm{~s}$ in a fix point $P$.

Physical filter parameters (absorption and diffusion) were estimated using [13]. Since only 2D temperature maps were available, a 2D implementation of the EKF was employed. Finally, the EKF was compared with a matched Kaiser Bessel FIR.

A second HIFU heating was performed $20 \mathrm{mn}$ later (once heat evacuation of the first experiment was completed) using a modified heating protocol. Here, the focal point position was electronically updated each TR/4 ms in four different locations $( \pm 0.5 \mathrm{~mm}$ in each axis from the point $P$ ). The AEKF was then applied, using the BHTE configuration obtained from the first fixed point experiment (which is thus not adapted to the present case), in order to observe its performance with a deliberately mis-configured BHTE model

b) Heating experiment on an in-vivo porcine kidney: MRI guided HIFU heating was performed in vivo in the kidney of a pig under general anesthesia. Since the kidney was static during the experiment, no focal point position adjustment was required. Dynamic MR-temperature imaging was realized in the Philips Achieva 1.5 T MRI and employed sequence was configured as follows: 1500 dynamic sagittal images, one slice, $\mathrm{TR}=127 \mathrm{~ms}, \mathrm{TE}=25 \mathrm{~ms}$, flip angle $=35^{\circ}, \mathrm{FOV}=142.5 \times 285$ $\mathrm{mm}^{2}$, voxel size $=3 \times 3 \times 6 \mathrm{~mm}^{3}$. Heating was performed using 250 Watts of electrical power during a period of 19 s. After completion of the experiments the animals were euthanized by intravenous injection of Pentobarbital. The animal experiments were conducted in agreement with the french law on animal experimentation and in compliance with institution's rules for animal care and use.

\section{RESULTS}

\section{A. Simulations}

Figure 1 shows the filter performances on a simulated dataset. The FIR filter (figure 1a) introduces a latency inherent to its design, while the EKF (figure 1b,c) avoids this effect. When configured with the exact physical parameters (figure 1b) the filter does not introduce any bias on accuracy and improves the output precision. An emphasis on the measured data $(\mathrm{Q}=10)$ leads to a little smoothing of the temperature. At the contrary, a high confidence in the model $(Q=0.1)$ provides an efficient noise removal in this case. Figure 1c shows the influence of an imperfect configuration of the model (in this case with an $50 \%$ overestimated absorption coefficient). Here, a bias on accuracy is observed, especially for $Q$ values giving a large influence to the model. On the other hand, using small values of $Q$ reduces the penalty on the accuracy but limits the filter ability to reduce measurement noise.

The error bias introduced by an approximative configuration of the BHTE based model was then investigated using a 3D implementation of the EKF. With the employed absorption and

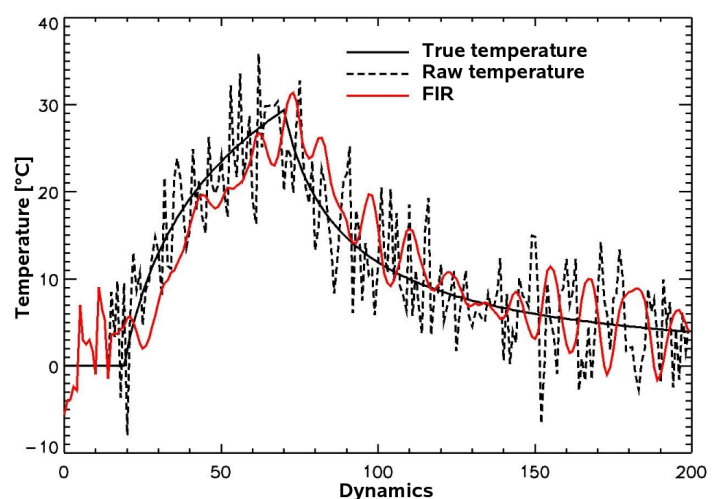

(a)

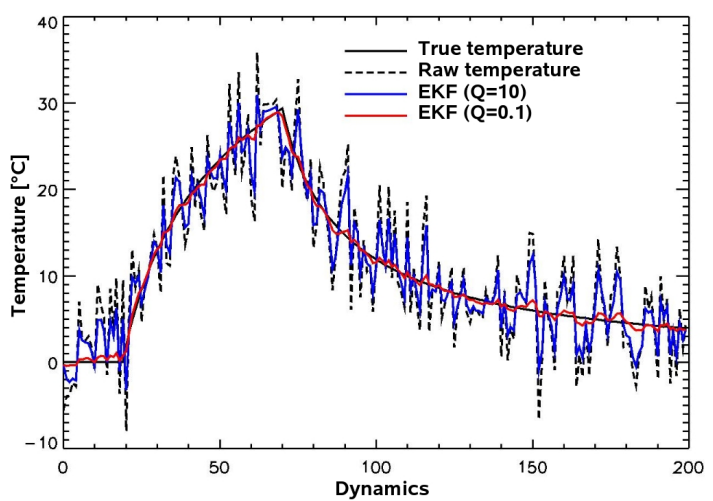

(b)

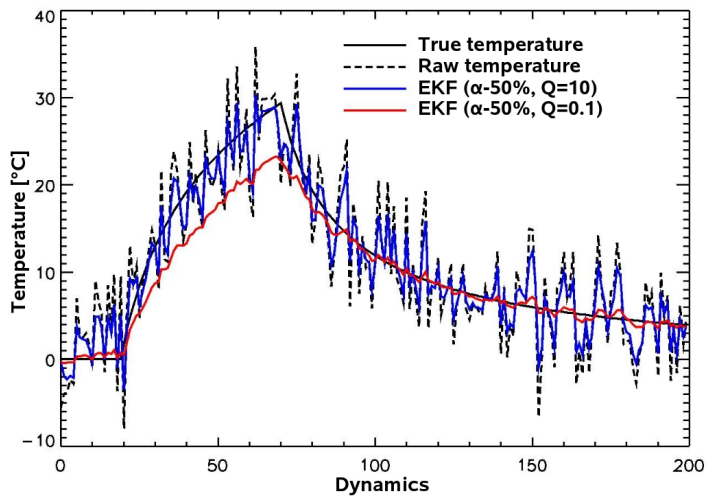

(c)

Fig. 1. Examples of temporal filtering obtained in the focal point of the simulated dataset: results obtained using (a) a FIR filter (a) an EKF filter configured with the true absorption and diffusion values $(\alpha=0.2$ and $D=1 \mathrm{e}$ 7 ), (c) an EKF filter badly configured (wrong absorption $(\alpha=0.1)$ with true diffusion $D=1 \mathrm{e}-7)$.

diffusion coefficients and the chosen spatial resolution of the simulation, the choice of the absorption coefficient has a larger impact than the choice of the diffusion coefficient. Therefore, an illustration of the bias introduced by an approximative configurations of the BHTE based model, obtained with several incorrect aborption values, is reported in figure 2 . For this, the filter was tested using different $Q$ values over all $\mathrm{N}$ datasets with an absorption coefficient varying for $-50 \%$ to $+50 \%$ of its true value. Evaluation of the error bias introduced by a misconfiguration of the absorption coefficient are plotted in figure 


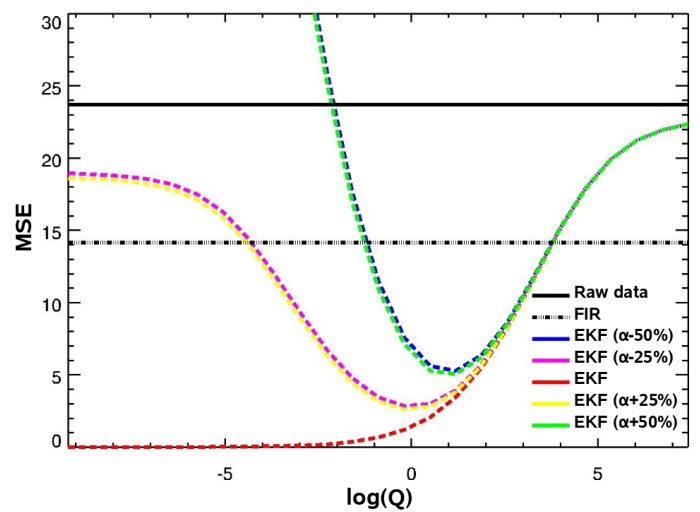

(a)

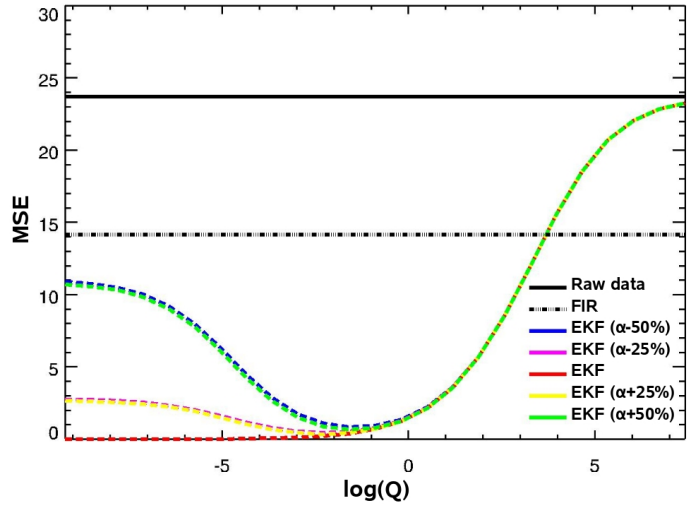

(b)

Fig. 2. Mean squared error of the filtered signal obtained with a 3D implementation of the EKF filter in the focal point of the simulated dataset. Influence of absorption on the mean square error (MSE) of the filtered temperature curve during heat-up (a) and cool-down (b) periods.

2a,b. It can be observed that optimal $Q$ values are different for the heating period (figure 2a,) and for the cooling period (figure 2b) that illustrates the time varying accuracy of the BHTE model. However, for all configurations, even in the case of a mis-configuration of the BHTE model with a bias of \pm $50 \%$ of the input absorption coefficient, a good choice of the $Q$ value can guarantee a better filter performance compared to the FIR filter. Therefore, an adaptive tuning of $Q$ during the intervention appears mandatory.

The performances of the presented adaptive EKF was investigated and the obtained filtered temperature with the time optimized $Q$ values are plotted in figure 3. The AEKF used a 3D implementation of the model that was configured with an incorrect absorption coefficient of $-50 \%$ of its true value and with the true diffusion coefficient. These configuration is thus similar to the EKF configuration employed in figure $1 \mathrm{c}$ and results are thus directly comparable. The initial mean square errors of $22.7^{\circ} \mathrm{C}$ and $21.9^{\circ} \mathrm{C}$ during the heat up and cool down periods were reduced to $8.1^{\circ} \mathrm{C}$ and $0.5^{\circ} \mathrm{C}$ using the mis configured AEKF. The same configuration of the EKF lead to a MSE of $26.9^{\circ} \mathrm{C}$ and $1.2^{\circ} \mathrm{C}$ with $\mathrm{Q}=0.1$ and $8.4^{\circ} \mathrm{C}$ and $8.5^{\circ} \mathrm{C}$ with $\mathrm{Q}=10$ (see figure $2 \mathrm{c}$ ) for heating and cooling periods respectively. Therefore, AEKF performances are similar to EKF with $Q=10$. during heating and similar to EKF with

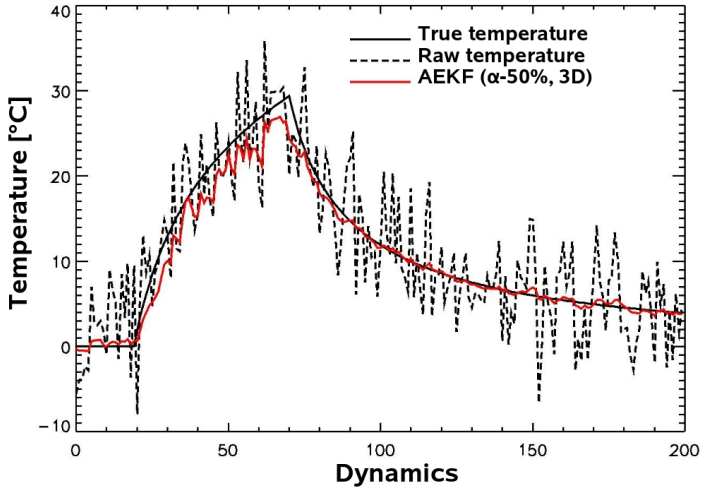

(a)

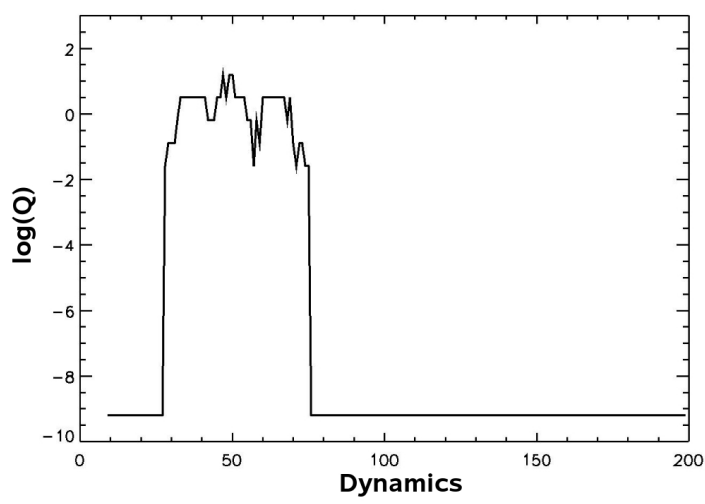

(b)

Fig. 3. Effects of an adaptive EKF using a 3D implementation of the BHTE model with a mis configured absorption coefficient (with $\alpha=0.1$ which represents $-50 \%$ of its true value) and the true diffusion coefficient (similar configuration to the EKF in figure 1c). Filtered temperature curve obtained with the AEKF is shown in (a) and the time optimized $Q$ values are reported in (b). Compared to the FIR ourput (figure 1a), the latency was substancially reduced. Then, before heating and during cooling, where the BHTE model was properly configured, the AEKF provided a better noise reduction compared to the FIR or the EKF with $\mathrm{Q}=10$ (figure 1c) However, the AEKF depicted similar performances to the $\mathrm{EKF}$ with $\mathrm{Q}=0.01$ (figure 1c, where an emphasis on the model is given). On the contrary, during the heating period, when the BHTE model was incorrectly configured, the mean sqaure error (MSE) obtained with the tested filters (FIR: $18.2^{\circ} \mathrm{C}$, EKF with $\mathrm{Q}=0.1: 26.9^{\circ} \mathrm{C}$ ) was reduced to $8^{\circ} \mathrm{C}$ with the AEKF that provides comparable results to the EKF with $\mathrm{Q}=10\left(\mathrm{MSE}=8.4^{\circ} \mathrm{C}\right.$, where an emphasis on measurement is given).

$Q=0.1$ during cooling confirming the benefit of an adaption of the $Q$ value. Finally, the AEKF clearly outperforms the FIR that provides a MSE of $18.3^{\circ} \mathrm{C}$ and $11.8^{\circ} \mathrm{C}$ for each of the two periods (see figure 2a).

Since, the BHTE based model was only incorrect during the heat up period (since absorption is only relevant during power delivering). The time optimized $Q$ values increased during this period in order to give more confidence in the measurements and converged into an interval of $[\log (0), \log (2)]$ (figure $1 b$ ). This corresponds well to the optimum $Q$ values (global minimum) obtained in figure $2 \mathrm{a}$ with the blue curve $(-50 \%)$. Then, the model being true during the cooling down period, the optimized $Q$ values decreased to improve the confidence in the model.

The potential of the proposed AEKF to automatically adapt and optimize the value of $Q$ was tested over the same datasets 


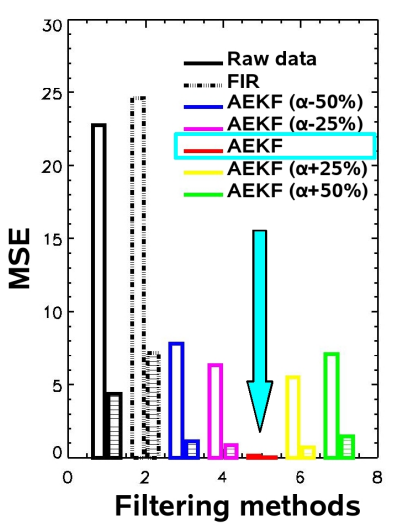

(a)

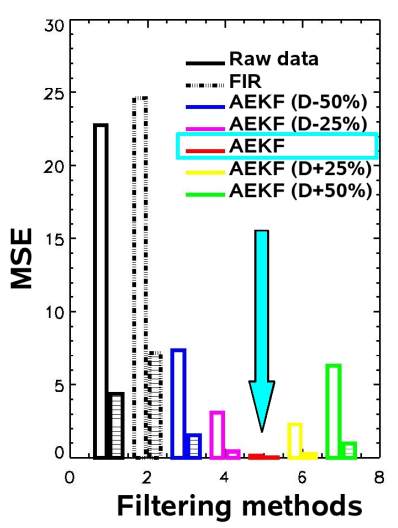

(c)

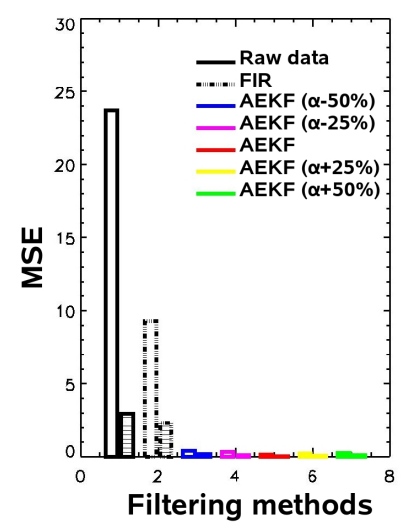

(b)

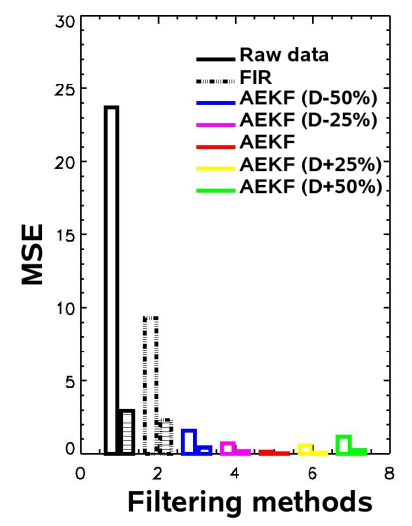

(d)

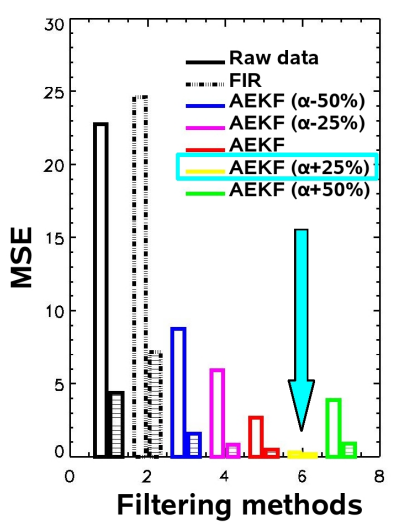

(a)

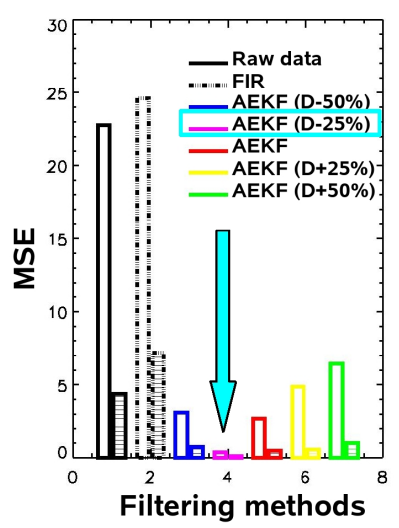

(c)

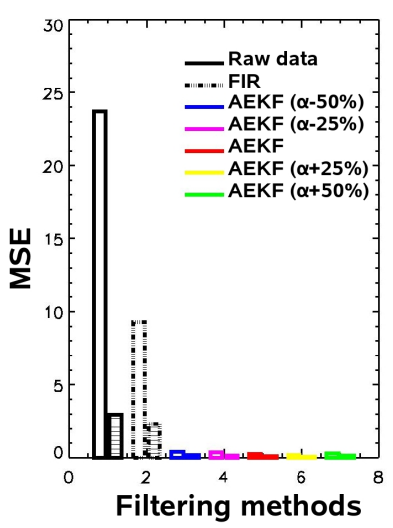

(b)

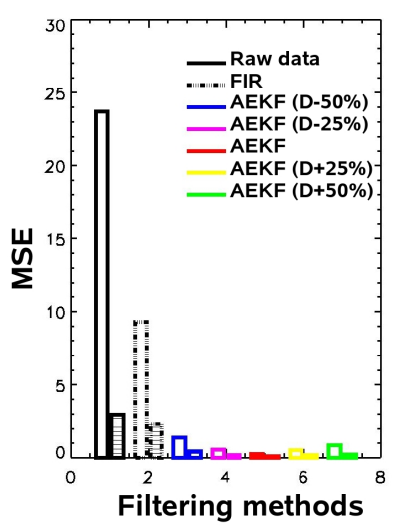

(d)

Fig. 4. Temporal filtering performance obtain using several configurations of the model using a 3D implementation of the filter. Influence of absorption $(a, b)$ and diffusion (c,d) on the mean square error (MSE) of the filtered temperature curve during heat-up $(\mathrm{a}, \mathrm{c})$ and cool-down $(\mathrm{b}, \mathrm{d})$. The AEKF achieves a better filter performance than the FIR even if the tissue absorption $\alpha$ or the thermal diffusion are deliberately miss-configured by $\pm 50 \%$. For each method, the MSE average and standard deviation over the $\mathrm{N}$ datasets are plotted in the empty box and the dashed box respectively.

and the results are plotted in figure 4 . The influence of a mis configuration of both absorption (4a,b) and diffusion $(4 \mathrm{c}, \mathrm{d})$ coefficients was evaluated during the heat-up $(4 a, c)$ and cooldown $(4 \mathrm{~b}, \mathrm{~d})$ periods. The resulting accuracy obtained with the FIR filter was comparable to the accuracy without filtering, which is mainly due to the latency introduced by the filter and its inability to follow rapid temperature variation. However, during the cool down period, the FIR allows to improve the resulting accuracy by a factor of 3 , due to the reduced temperature variation between two successive temperature points (compared to the heat up period). Using the AEKF, the resulting accuracy was reduced by a factor in the worst tested case by a factor 3 and 15 for the heat up and cool down period respectively. Therefore, the AEKF allows to outperform the FIR performances even in the case of a mis-configuration of the BHTE model with a bias of $\pm 50 \%$ of the input parameters.

The influence of a 2D implementation of the filter was then evaluated over the same simulated datasets as for the 3D AEKF evaluation. Figure 5 shows the impact of a mis

Fig. 5. Influence of a 2D implementation of the filter: Similarly to figure 4 the impact of an incorrect absorption (a,b) and an incorrect diffusion $(\mathrm{c}, \mathrm{d})$ was evaluated during both heat up $(\mathrm{a}, \mathrm{c})$ and cool donw $(\mathrm{b}, \mathrm{d})$ periods. Again, the AEKF achieves a better performance than the FIR even with a 2D implementation of the filter and when the tissue absorption $\alpha$ or the thermal diffusion are deliberately miss-configured by $\pm 50 \%$. However, an over estimation of the absorption (by $+25 \%$ of the true value) or an under estimation of the diffusion coefficient (by $-25 \%$ of the true value) that help to compensate for the neglected inflow from adjacent slices lead to improved performances compared to a true configuration

configuration of both absorption (5a,b) and diffusion $(5 \mathrm{c}, \mathrm{d})$ coefficients during the heat-up $(5 \mathrm{a}, \mathrm{c})$ and cool-down $(5 \mathrm{~b}, \mathrm{~d})$ periods. Here, the best performances of the AEKF are not achieved with the true configuration of the absorption and diffusion coefficients. Here, the focal point size in the third dimension is $7.88 \mathrm{~mm}$ which is three time larger that the resolution of the simulation in the third dimension. In these conditions, all energy supposed to be delivered above and below the 2D single slice is not considered with this $2 \mathrm{D}$ implementation. Therefore, an AEKF with either an absorption coefficient over estimated of $+25 \%$ of the true value or a diffusion coefficient under estimated of $-25 \%$ of its true value allows to compensate for the lack of received energy and in this case outperform an AEKF with a true configuration. Similarly to the 3D AEKF evaluation, the resulting accuracy was reduced by a factor in the worst tested case by a factor 3 and 15 for the heat up and cool down period respectively. Therefore, the AEKF allows to outperform the FIR performances even 
with a 2D implementation (with the employed conditions) in the case of a mis-configuration of the BHTE model with a bias of $\pm 50 \%$ of the input parameters.

\section{B. Heating experiment on an agarose gel phantom}

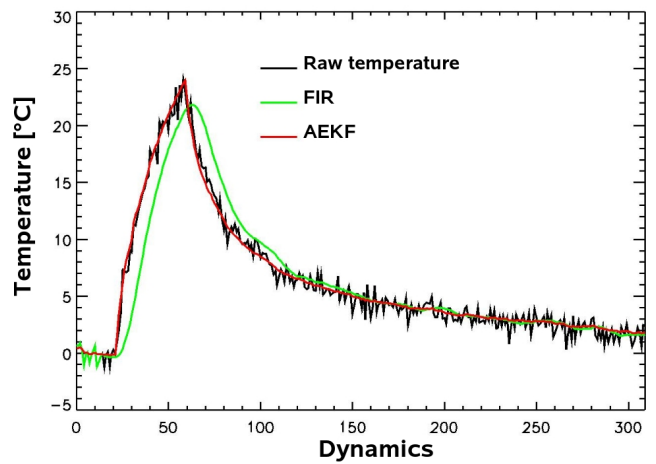

(a)

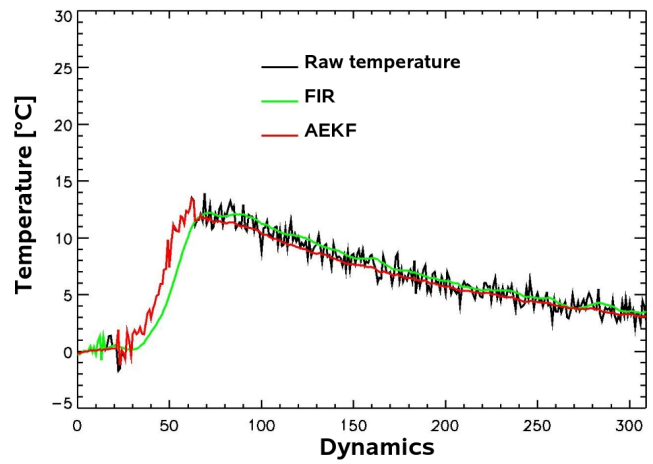

(b)

Fig. 6. Filtering performances of a FIR and the AEKF obtained on an agarose gel during heating experiments. (a) temperature evolution obtained during the first heating experiment in the focal point using a properly configured BHTE based model. (b) temperature evolution in the same point during a the second heating experiment where four different locations where iteratively heated. The AEKF follows measurements during heat up period since the model is incorrect but gains confidence during cool down period where the BHTE based model is correct.

The method was evaluated on an agarose gel phantom during a HIFU heating experiment (see figure 6). The AEKF provided a better reduction of the temperature noise and did not introduce large latency as obtained with the FIR (see figure 6a). A second heating experiment was then realized with a different heating protocol where the focal point position was electronically updated in four different locations. The AEKF was run using the same configuration as in the fixed point experiment, which is thus expected to be incorrect for this experiment. Results are reported in figure $6 \mathrm{~b}$ and shows that the AEKF considers the model as incorrect during the heating period where the filter has a low confidence in the model. During the cooling period, the model is correct since only the heat diffusion is present (and is properly configured) and the filter stronly improves its confidence in the BHTE based model that result in a substancial reduction of the temperature noise. Again, the bias introduced by the latency of the FIR is also avoided with the AEKF.

\section{Heating experiment on an in-vivo porcine kidney}

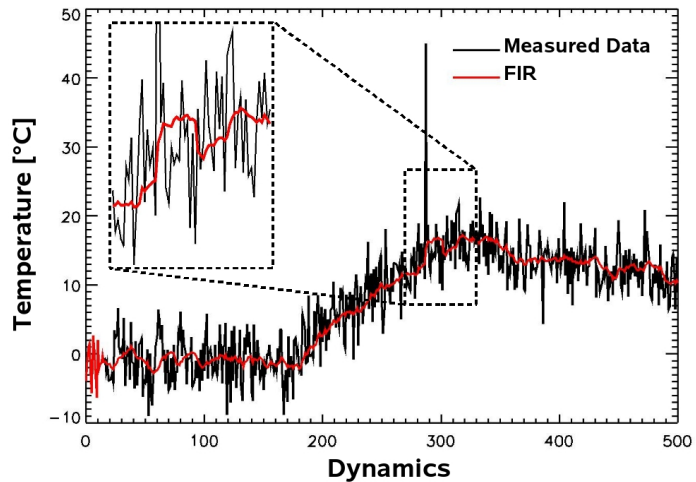

(a)

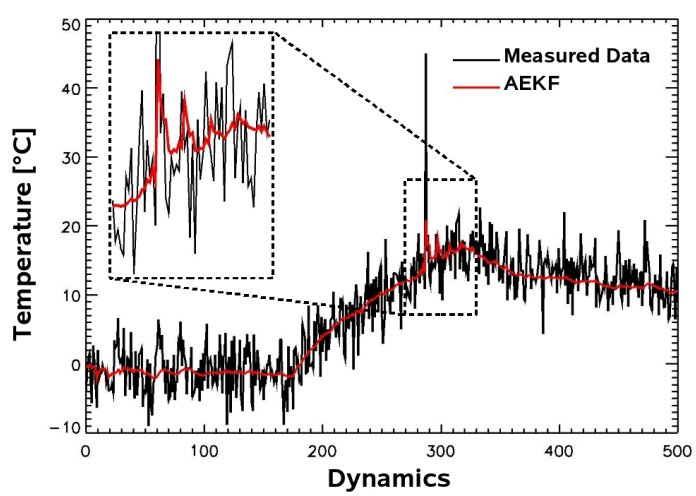

(b)

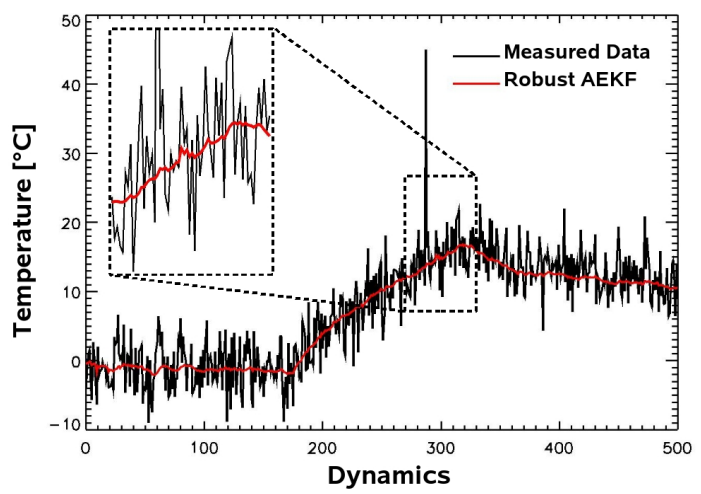

(c)

Fig. 7. Temperature evolution of the HIFU experiment on an in-vivo porcine kidney using a (a) FIR filter, (b) the AEKF, (c) the robust AEKF. The robust AEKF allows to detect this strong temperature artifact as outlier and to replace it by the BHTE model prediction value.

An in-vivo heating experiment was finally performed in a porcine kidney. A temperature elevation of $18^{\circ} \mathrm{C}$ was reached in the focal point. A large temperature artifact $\left(45^{\circ} \mathrm{C}\right)$ was simulated at the $288^{\text {th }}$ dynamic in the focal point. This type of artifact induced a strong perturbartion of both the FIR filter (figure 7a) and the AEKF (figure 7b). The robust AEKF approach allowed to detect this artifacted datapoint and the resulting filtered temperature was not affected (figure 7c). 


\section{Real time benchmarking}

The computation time of the EKF (using a dual processor, INTEL $3.1 \mathrm{GHz}$ Penryn, two cores, $8 \mathrm{~GB}$ of RAM) was 0.71 $\mathrm{ms}$ for a $2 \mathrm{D}$ image of resolution $32 \times 32$ and $11.4 \mathrm{~ms}$ for a $3 \mathrm{D}$ image of resolution $32 \times 32 \times 16$. In the AEKF, the additional time required for the autocalibration of the $Q$ value is defined by the number of steps toward the optimum $Q$ value. In our approach, an upper bound was imposed to the algorithm and was defined in order to ensure the real time constraint available in any case. For the case, of HIFU experiment where a TR of $100 \mathrm{~ms}$ was employed with a slide window of 10 images, the maximum number of iteration toward convergence was empirically fixed to 12 (since $12 \times 10 \times 0.71 \mathrm{~ms}=85.2 \mathrm{~ms}<$ $T R$ ) to guarantee the real time condition.

\section{Discussion}

\section{A. BHTE model based temperature prediction}

The performance of the BHTE model relies primarily on the accurate representation of the physical heating process. For this, it requires a priori knowledge of the exact form of the focal point $P_{(\vec{r}, t)}$ and the local absorption and diffusion coefficient $\alpha$ and $D$. In particular, the choice of the later two coefficients is in practice problematic, since it either requires empirical data or a suitable calibration experiment. This is complicated by the fact that the spatial resolution of typical MR-sequences for interventional imaging are of the same order than the observed diffusion phenomena and focal point sizes, which leads to partial volume effects [14]. Therefore, a calibration results only in apparent absorption and diffusion coefficients, which will vary with increasing voxel sizes. Furthermore, although both apparent coefficients can be expected to be sufficiently homogeneous within large organs such as the liver, this assumption could break down in smaller heterogeneous organs such as the kidney, or close to organ boundaries, where both become spatially variant. Another limitation of the predictor model arises from the negligence of the heat evacuation due to tissue perfusion. Although this is for the presented experiment on a gel of no consequence, future work on in-vivo tissue requires a careful assessment of the corresponding error bias and potentially to take the perfusion term in equation 4 into account.

In addition, since the achievable MR-acquisition time severely limits the available volume coverage, the BHTE has to be in practice applied to $2 \mathrm{D}$ or severely undersampled $3 \mathrm{D}$ datasets. As shown in figure 5 the influence of a $2 \mathrm{D}$ implementation of the model is of little consequence as long as the spatial resolution in slice direction remains much larger than the characteristic diffusion length at the given temporal resolution of the MR-sequence.

Finally, the temperature prediction model is also directly influenced by the choice of the size (in $\mathrm{mm}$ ) of the prediction area. A large area could prevent the real time use of the method (especially for the autocalibration that requires several temperature prediction to adjust the $Q$ parameter). On the other hand, a small area may be insufficient to encompass the whole heated area introducing undesirable high frequency in the Fourier domain that could be further reduced using apodization function. In the presented gel and in-vivo experiments under typical condition of clinical ablation, an area of $32 \times 32$ pixels with a pixel size in the range of a milimeter was found to be a good compromise.

\section{B. Autocalibration performances of the EKF, Dynamic evalu- ation of the BHTE model accuracy}

Although the BHTE model is an efficient way to predict the temperature, the uncertainty of the model is a priori not known and was shown to vary over time (especially between heating up and cooling down period). The autocalibration of the $Q$ parameter allows to correctly handle the model accuracy in the proposed filter. Nevertheless, the autocalibration process is influenced by two parameters: the spatial window size $(M)$ and the temporal window size $(N)$ employed to evaluate the filter accuracy (see in equation 7).

The spatial window size has to cover voxels contained in the ablation area and thus subject to the influence of the heat absorption and the heat diffusion. In our experiment, 9 voxels around the focal point were selected for 2D experiments and 9 voxels of each of the two adjacent slices were included for $3 \mathrm{D}$ experiments (leading to a total of 27 voxels). Although the number of voxels prone to temperature increase can be limited in a fixed point heating experiment, this issue is expected to be clearly reduced for volumetric ablation (leading to a larger ablated area).

The temporal window size directly influences the frequency response of the proposed adaptation of $Q$. A large temporal window leads to stable convergence of the $Q$ value, while introducing a latency on the $Q$ adaptation. A small temporal window allows reactive adaptation of $Q$ at the price of a reduced statistical sample contributing to equation 7 . Consequently, this parameter has to be adjusted depending on the temporal dynamic of the temperature between two successive acquisitions. The auto-adaptation of $Q$ allows to dynamically find the smallest $Q$ value that maintains the introduced error bias of temperature below a predefined threshold defined by the $\varepsilon$ parameter. This allows maximal measurement noise filtering under the condition of a guaranteed accuracy. For the case of a severely mis-configured filter, the auto-adaptation will gives a higher emphasis of the measured data (high value of $Q$ ) in order to limit the introduced accuracy bias. In practice this means the filter will be less efficient for noise removal, however, a severely mis-configured filter will not introduce systematic errors in the filtered data. ited to an upper bound defined by the $\varepsilon$ parameter.

Contrary to autocalibration approaches directly based on the analysis of the innovation sequence such as in [15], the proposed adaptation allows a further reduction of the temperature noise at an accuracy cost, which is bounded by the predefined maximal accepted penatly of acuracy $\varepsilon_{\text {threshold }}$.

\section{Robust AEKF approach}

In general, artifact detection is complicated by the requirement to find a robust criterion allowing to differentiate between a measurement artifact and changes due to the dynamic of the measured process. Since the proposed AEKF formalism 
employes the BHTE as a physical model for data prediction of the heating process, it is straightforward to seamlessly integrate a robust outlier detection without significant additional computational overhead. This is achieved by examining the innovation $S_{t}$ for unphysical changes and to replace unphysical measuements by the predictor value $T_{t}^{-}$. Therefore, contrary to temporal filtering, where the amplitude of the artifacted datapoint still influences the amplitude of the filtered data (figure $7 \mathrm{a}, \mathrm{b}$ ), the proposed approach allows to replace the value entirely with a prediction based on the physical heating model (figure $7 \mathrm{c}$ ). Furthermore, the proposed AEKF filter also accounts for the variations of the average model error during the heating process and thus conviently allows to adjust the outlier rejection criterion by using the updated $\varepsilon(t-1)$ and $\sigma\left(S_{t-1}\right)$ values.

\section{Clinical aspects of temporal filtering of the temperature}

The presented data has shown that due to the use of a physical model of the heating process, the AEKFs model based filtering performance surpasses the results of more simple filter designs. However, in order to evaluate the usefullness of the proposed AEKF for clinical applications, two other categories of requirements have to be considered: Patient safety aspects and real-world practicability. Since the proposed filter is intended for real-time MR-guidance of non-invasive HIFU ablations, additional latency and accuracy degradation can directly affect the success of the intervention and ultimately patient safety. Therefore, despite the fact that the BHTE model parameters are prior to the intervention only available as coarse estimates, the AEKF must be able to cope with a severe misconfiguration without impairing the accuracy of the filtered temperature values, as shown in figure $1 \mathrm{c}$ for the EKF. The AEKF filtering process was designed so that even a worst case scenario merely results in an ineffective noise removal, without systematic errors introduced by the filter as shown in figure 3 a and $6 \mathrm{~b}$. Furthermore, reliable thermal dose measurements are frequently hampered by the effects of occasional spontaneous motion events or instabilities/errors of the employed realtime data processing. The proposed integrated outlier detection reliably identifies and removes such temperature artifacts and thus allows to perfom sustained dosimetry over extended durations of several minutes. Since the artifacted datapoint is entirely replaced by a model based estimate, a systematic error bias of the final thermal dose value is prevented.

The proposed filter design is more complex than nonadaptive FIR or IIR filter designs, as a consequence the second important aspect is the practicality of it's use. The main disadvantage of the proposed BHTE based AEKF is that the filter requires an approximate estimate of the absorption coefficient $\alpha$ and the diffusion coefficient $D$ prior to the intervention. Both can be provided on an individual basis based on a low-power test shot as shown in [13]. Alternatively, since an adequate filter performance does not require the exact knowledge of either parameter, for standardised clinical scenarios such as uterine fibroid ablations, both values could be supplied based on averaged patient data. All other parameters of the filter are autocalibrated during the intervention, which reduces the risk of an arbitrary/empirical calibation by the user. Finally, although the AEKF implementation has a higher computational overhead than FIR or IIR designs, the benchmarking results show that the design is entirely compatible with the requirement of interventional guidance of high-framerate imaging associated with low processing latencies.

\section{CONCLUSION}

The proposed autocalibrated extended Kalman filter based on the bio-heat transfer equation was demonstrated to improve both precision and accuracy of MR-thermometry compared to simpler filter designs such as FIR-filters, without introducing undesired latency. However, the increased complexity of a model based filter design also increases the risk of systematic errors for the case of severe mis-configuration. For clinical applications in the field of therapy guidance this would represent an unacceptable risk, due to the fact that the filtered data is used for direct retroactive feedback control, and concequently errors can not be undone retrospectively. Since this directly influences patient safety, the proposed autocalibrated extended Kalman filtering formalism was used to control and limit the impact of the filter on latency and accuracy during the entire intervention. In conjunction to the additional outlier rejection algorithm, which replaces sparse temperature measurement artifacts with BHTE prediction based estimates, this represents a promising approach to improve the precision and the feasability of extended MR-thermometry and dosimetry for MR-guidance of HIFU ablations under clinical conditions.

\section{REFERENCES}

[1] H. Laumonier, J. Blanc, B. Quesson, O. Seror, C. Laurent, P. BioulacSage, C. Balabaud, and H. Trillaud, "Real-time monitoring of hepatocellular carcinoma radiofrequency ablation by quantitative temperature MRI," Semin Liver Dis, vol. 26, pp. 391-39, 2006.

[2] R. Puls, S. Langner, C. Rosenberg, K. Hegenscheid, J. Kuehn, K. Noeckler, and N. Hosten, "Laser ablation of liver metastases from colorectal cancer with MR thermometry: 5-year survival." J Vasc Interv Radiol., vol. 20, no. 2, pp. 225-34, 2009.

[3] H. Cline, J. Schenck, K. Hynynen, R. Watkins, S. Souza, and F. Jolesz, "MR-guided focused ultrasound surgery," J Comput Assist Tomogr, vol. 16, no. 6, pp. 956-965, 1992.

[4] C. Mougenot, B. Quesson, B. de Senneville, P. de Oliveira, S. Sprinkhuizen, J. Palussière, N. Grenier, and C. Moonen, "Threedimensional spatial and temporal temperature control with MR thermometry-guided focused ultrasound (MRgHIFU)," Magn Res Med, vol. 61 , no. 3, pp. 603-14, 2009.

[5] S. Sapareto and W. Dewey, "Thermal dose determination in cancer therapy," Int. J. Radiation Oncology Biol. Phys., vol. 10, pp. 787-800, 1984.

[6] T. Conturo and G. Smith, "Signal to noise in phase angle reconstruction : Dynamic range extension using phase reference offsets," Magnetic Resonance in Medicine, vol. 15, pp. 420-437, 1990.

[7] S. Roujol, M. Ries, B. Quesson, C. Moonen, and B. Denis de Senneville, "Real-time MR-thermometry and dosimetry for interventional guidance on abdominal organs," Magnetic Resonance in Medicine, vol. 63, no. 4, pp. 1080-1087, 2010.

[8] R. Kalman, "A new approach to linear filtering and prediction problems," Journal of Basic Engineering, vol. 82, no. 1, pp. 35-45, 1960.

[9] J. Potocki and H. Tharp, "Concurrent hyperthermia estimation schemes based on extended Kalman filtering and reduced-order modelling," Int J Hyperthermia, vol. 9, no. 6, pp. 849-65, 1993.

[10] H. Pennes, "Analysis of tissue and arterial blood temperatures in the resting human forearm," Journal of applied physiology, vol. 1, no. 2, pp. 93-122, 1948 . 
[11] G. Ye, P. Smith, and J. Noble, "Model-based ultrasound temperature visualization during and following HIFU exposure," Ultrasound Med Biol, vol. 36, no. 2, pp. 234-49, 2010.

[12] A. Chauvenet, A manual of spherical and practical astronomy, 1st ed. Philadelphia: Lippincottt, 1863.

[13] D. I., P. de Oliveira, C. Laurent, C. Mougenot, N. Grenier, C. Moonen, and B. Quesson, "Non-invasive determination of tissue thermal parameters from high intensity focused ultrasound treatment monitored by volumetric MRI thermometry," NMR Biomed, vol. 22, no. 8, pp. 843$51,2009$.

[14] N. Todd, U. Vyas, J. de Bever, A. Payne, and D. Parker, "The effects of spatial sampling choices on mr temperature measurements," Magnetic Resonance in Medicine, vol. 65, pp. 515-21, 2011.

[15] R. Mehra, "On the identification of variances and adaptive kalman filtering," Automatic Control, IEEE Transactions on, vol. 15, no. 2, pp. $175-184,1970$ 